\title{
Small noncoding RNA GcvB is a novel regulator of acid resistance in Escherichia coli
}

\author{
Ye Jin ${ }^{1}$, Rory M Watt ${ }^{2}$, Antoine Danchin ${ }^{3}$ and Jian-dong Huang*1
}

Address: ${ }^{1}$ Department of Biochemistry, Li Ka Shing Faculty of Medicine, University of Hong Kong, Pok Fu Lam, Hong Kong SAR, PR China, ${ }^{2}$ Oral Biosciences, Faculty of Dentistry, University of Hong Kong, Pok Fu Lam, Hong Kong SAR, PR China and ${ }^{3}$ Genetics of Bacterial Genomes, CNRS URA2171, Institut Pasteur, 28, rue du Docteur Roux, Paris Cedex 15, 75724, France

Email: Ye Jin - yejin@hkucc.hku.hk; Rory M Watt - rmwatt@hku.hk; Antoine Danchin - antoine.danchin@normalesup.org; Jiandong Huang* - jdhuang@hkucc.hku.hk

* Corresponding author

Published: 20 April 2009

BMC Genomics 2009, 10:165 doi:10.1/86/147|-2164-10-165

This article is available from: http://www.biomedcentral.com/I47I-2/64/I0/I65

(C) 2009 Jin et al; licensee BioMed Central Ltd.

This is an Open Access article distributed under the terms of the Creative Commons Attribution License (http://creativecommons.org/licenses/by/2.0), which permits unrestricted use, distribution, and reproduction in any medium, provided the original work is properly cited.
Received: 26 January 2009

Accepted: 20 April 2009

\begin{abstract}
Background: The low pH environment of the human stomach is lethal for most microorganisms; but not Escherichia coli, which can tolerate extreme acid stress. Acid resistance in $E$. coli is hierarchically controlled by numerous regulators among which are small noncoding RNAs (sncRNA).

Results: In this study, we individually deleted seventy-nine sncRNA genes from the E. coli KI2MGI655 chromosome, and established a single-sncRNA gene knockout library. By systematically screening the sncRNA mutant library, we show that the sncRNA GcvB is a novel regulator of acid resistance in $E$. coli. We demonstrate that $\mathrm{GcvB}$ enhances the ability of $E$. coli to survive low pH by upregulating the levels of the alternate sigma factor RpoS.
\end{abstract}

Conclusion: GcvB positively regulates acid resistance by affecting RpoS expression. These data advance our understanding of the sncRNA regulatory network involved in modulating acid resistance in $E$. coli.

\section{Background}

The ability to survive the extremely low $\mathrm{pH}$ environment present within the stomach ( $\mathrm{pH}$ values $<2.5)$ is essential for colonization of the intestine by commensals and for the pathogenesis of enteric bacteria. Three distinct acid resistance (AR) systems are responsible for protecting bacterial cells from acid shock [1-3]. AR system 1 is a glucoserepressed system and does not require any external amino acids to function at low $\mathrm{pH}[1]$. Unidentified components of this system are controlled by the alternate sigma factor RpoS, with the underlying mechanism remaining unclear [1]. In contrast, AR systems 2 and 3 provide AR by consuming intracellular protons via the glutamate (AR2) and arginine (AR3) decarboxylation reactions [2,3]. AR2 is the most efficient AR system. It involves two isozymes of glutamate decarboxylase, encoded by genes gadA and $g a d B$, and a membrane-associated antiporter (GadC) that exchanges external glutamate for the intracellular decarboxylation product $\gamma$-aminobutyric acid (GABA) [2].

AR2 is hierarchically regulated by multiple regulators, which include small noncoding RNAs (sncRNA) DsrA and possibly GadY. DsrA activates the expression of $r p o S$ [4] and several AR genes including $h d e A B, \operatorname{gad} A X$, and gadBC [5]. GadY, whose effects on AR have yet to be determined, controls the synthesis of GadX and GadW, which are reg- 
ulators of the GadA and GadB glutamate decarboxylases $[6,7]$. In recent years, an increasing number of sncRNAs have been identified, but the functions of the vast majority of these are unknown. This prompted us to investigate whether sncRNAs in addition to DsrA and GadY, were involved in AR; and if so, to establish how they exerted their regulatory actions.

In the present study, seventy-nine snRNAs identified prior to 2006 [8-10] were selected for the construction of a single-gene knockout library within E. coli K12-MG1655. Comparison of each mutant with the wild type strain enabled us to identify the sncRNA GcvB as a previously unknown regulator of AR in E. coli. We demonstrate that GcvB positively regulates AR predominantly by upregulating the levels of the RpoS transcription factor (Sigma S). Our findings provide further insight into the regulatory roles played by sncRNAs in AR, which contributes to virulence of $E$. coli.

\section{Results}

\section{Construction of single-sncRNA gene knockout mutant collection in E. coli K-I 2 MGI655}

To systematically characterize sncRNAs, we established a single-sncRNA gene knockout library within E. coli K12MG1655. Recombineering techniques were used to replace the target gene with a selectable chloramphenicolresistance cassette, which was generated by polymerase chain reaction (PCR) using primers containing homology (45 nt) to the gene-flanking regions. This homologous recombination-based approach enabled precise gene deletion with minimal interference to adjacent genes (polar effects) [11,12]. In total, seventy-nine genes encoding sncRNAs identified prior to 2006 [8-10] were deleted individually from the MG1655 chromosome (see additional file 1).

\section{The sncRNA GcvB is a novel regulator of AR in E. coli}

We investigated the involvement of sncRNAs in the AR of $E$. coli by challenging the sncRNA mutants as well as the wild type strain by incubation in LB medium at $\mathrm{pH} 2.0$ (for 15 or 30 minutes), and comparing their percent survival and/or rates of recovery after $\mathrm{pH}$ neutralization (termed acid recovery). Unless otherwise stated, bacterial cells were grown for 5 hrs to the early stationary phase before the acid challenge. As expected, cells lacking DsrA, which is a known AR regulator, were sensitive to the acid challenge, confirming previous findings (Fig. 1A) $[4,5]$. However, deleting gadY did not affect acid survival (Fig. $1 \mathrm{~A})$, suggesting that GadY is not required for AR in spite of its positive control of GadX, a transcriptional activator of the GadA and GadB glutamate decarboxylases [6,7]. Interestingly, the absence of the sncRNA GcvB resulted in a significant reduction in acid survival (Fig. 1A, B). The same AR phenotypes in the sncRNA mutants were also
A

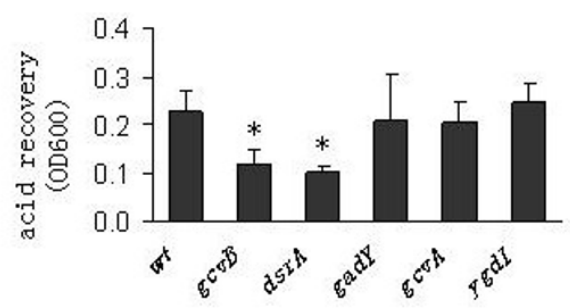

B
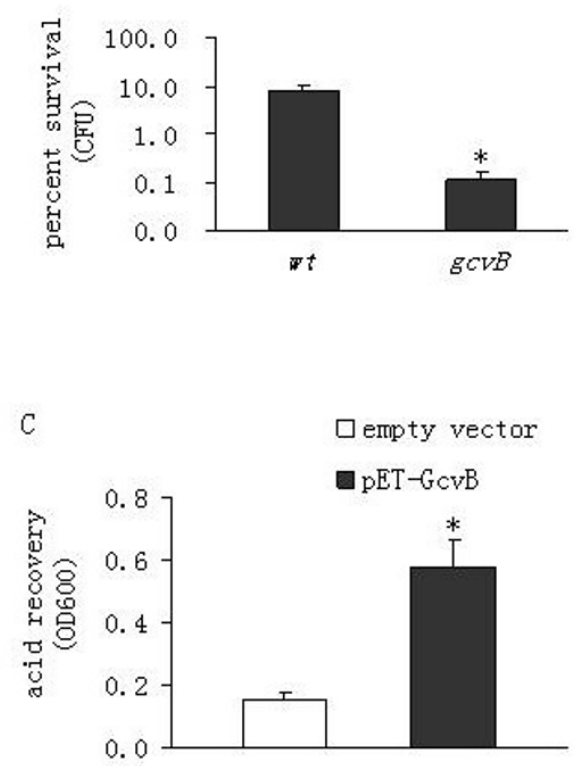

Figure I

Regulatory role of the small noncoding RNA GcvB in acid resistance in Escherichia coli. (A) MGI655-K/2 wild type strain (wt) and various deletion mutants were grown in Luria Broth broth $\left(\mathrm{pH} \mathrm{7.0)}\right.$ for $5 \mathrm{hr}$ at $37^{\circ} \mathrm{C}$. Cells were acid shocked at $\mathrm{pH} 2.0$ for $30 \mathrm{~min}$, and then neutralized by adding alkalinized LB ( $\mathrm{pH}$ 9.3). Cells were allowed to grow for an additional $3 \mathrm{hrs}$ before measurement of optical density at 600 $\mathrm{nm}$ (OD600). Two-tailed paired $\mathrm{t}$ tests were employed to compare each mutant with the wt in terms of acid resistance. (B) Because the gcvB mutant displayed a lower growth rate than the wt (data not shown), percent acid survival was determined to confirm the data of acid recovery measured as OD600. Cell viability was determined by plate counts and percent acid survival was calculated as the number of cfu remaining after the 30 min-acid treatment divided by the initial cfu at time zero (quoted as a percentage). (C) A plasmid expressing GcvB under the control of its native promoter was transformed into a gcvB mutant of $E$. coli MG I655. The mutant carrying an empty vector was used as a control. Cells were incubated for $5 \mathrm{hrs}$ before being acid challenged for 15 min. Error bars represent standard deviation $(* P$-value $<$ 0.05). 
observed during the exponential growth phase (data not shown), suggesting that GcvB exerts constant AR regulatory functions throughout the various growth phases. We also individually deleted $g c v A$ and $y g d I$ which are the two genes immediately adjacent to $g c v B$. Both mutants displayed comparable AR as the wild type (Fig. 1A). This shows that the observed acid vulnerability in the $\mathrm{gcvB}$ mutant is not due to the disruption of the activities of its adjacent genes.

Next, we cloned the gene encoding GcvB (together with its native promoter) into plasmid pET32a $(+)$ and overexpressed the sncRNA in the E. coli MG1655 gcvB null mutant. The $g c v B$ mutant carrying an empty vector served as a negative control. We found that overproducing $\mathrm{GcvB}$ restored the ability of the $g c v B$ mutant to survive at low $\mathrm{pH}$ (Fig 1C). These observations together with the knockout data revealed that GcvB acts as a positive regulator of AR.

\section{The known GcvB target OppA plays a minor, if any, role in GcvB-mediated AR}

GcvB has been demonstrated to repress the translation of $o p p A$ and $d p p A$ in $E$. coli by masking the ribosome binding sites of their mRNAs, which encode periplasmic substratebinding proteins of $\mathrm{ABC}$ uptake systems for amino acids and peptides [13-15]. To determine whether these two proteins played a role in GcvB-mediated AR, we individually deleted their encoding genes, and examined the ability of the resultant mutants to survive at low $\mathrm{pH}$. We found that deleting $o p p A$ slightly increased $A R$, as revealed by their acid recovery (OD600) and percent acid survival (cfu) levels, while the absence of $d p p A$ had little effect on AR (Fig. 2A, B). Thus DppA is not involved in GcvB-mediated $\mathrm{AR}$, and OppA plays a minor, if any, role in this process. We reasoned that the GcvB sncRNA may be exerting its effects on AR via a previously unidentified molecular target.

\section{GcvB mediates $A R$ by upregulating RpoS expression}

RpoS is the central regulator of glutamate-dependent AR2 genes such as $\operatorname{gadA}, \operatorname{gadBC}[1], \operatorname{gadX}[16], \operatorname{gadE}$ [17], and $\operatorname{gad} Y[6,7]$. It is also required for the AR1 system $[1,18]$. We therefore set out to determine whether GcvB regulated AR by modulating RpoS expression levels. We found that deleting $g c v B$ did not lead to a further reduction in acid survival levels in an rpoS mutant background (Fig. 3A). More importantly, the absence of $r p o S$ completely abolished AR elicited by the plasmid-encoded GcvB (Fig. 3B), suggesting that GcvB-mediated AR is dependent on RpoS. Next, an rpoS-lacZ translational fusion was constructed to examine whether the presence or absence of the GcvB sncRNA significantly affected $r p o S$ expression levels. Deleting $g c v B$ reduced the expression levels of the rpoSlacZ fusion by $50 \%$ (Fig. $4 \mathrm{~A}$ ), whereas $g c v B$ overexpression increased it 3-fold (Fig. 4B). Furthermore, deleting oppA,
A

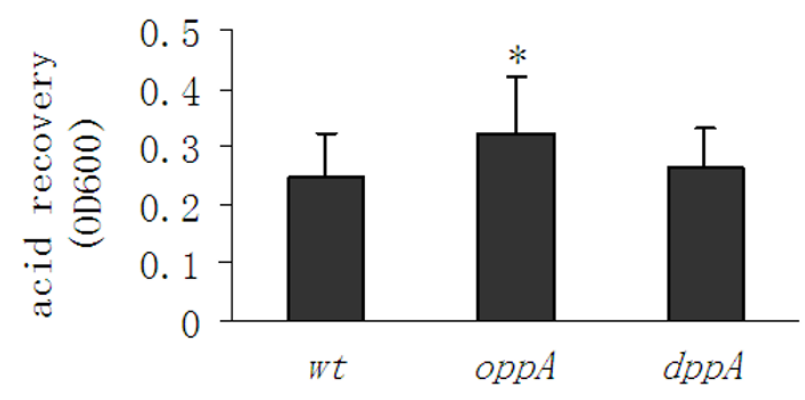

B

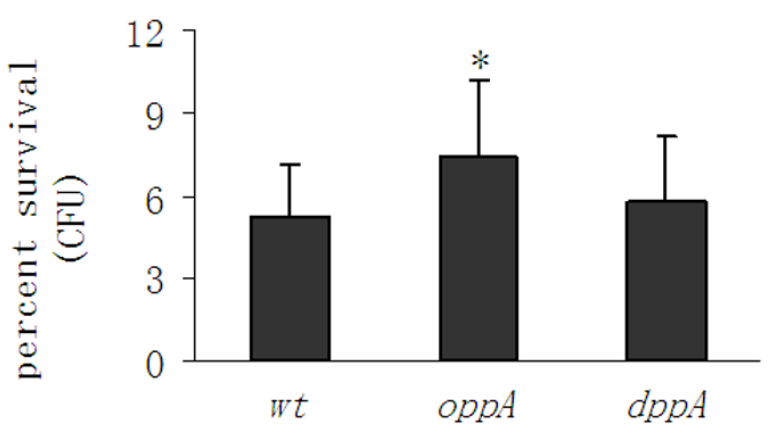

Figure 2

Effects of individually deleting oppA and $\triangle p p A$ on acid resistance in Escherichia coli. The acid-stress survival abilities of two individual deletion mutant strains lacking previously identified targets of GcvB were compared with those of the MGI 655 wild type (wt) strain. The abilities of strains to survive a 30 minute acid shock were quantified by two methods: $(A)$ acid recovery measured as optical density at $600 \mathrm{~nm}$ (OD600). (B) Percent survival determined by colony forming units (cfu). Error bars represent standard deviation $(* P$-value $<0.05)$.

whose absence had previously been shown to slightly increase AR levels, had no effect on rpoS-lacZ expression (Fig. 4C). This indicates that the GcvB-mediated regulation of RpoS expression does not involve OppA.

We then investigated whether GcvB indirectly controls RpoS production via an upstream regulator. In the literature, there are numerous reports outlining mechanisms by which the synthesis of RpoS is regulated at the transcriptional [19-22], translational and posttranslational levels [23-25]. Of these regulators of RpoS expression, Crp [20], H-NS [23], GadX [22], and a hybrid sensor kinase called BarA [21] have previously been associated with AR. Although BarA acts as a positive regulator of $r p o S$ transcription, its effect is restricted to the exponential phase. It 
A

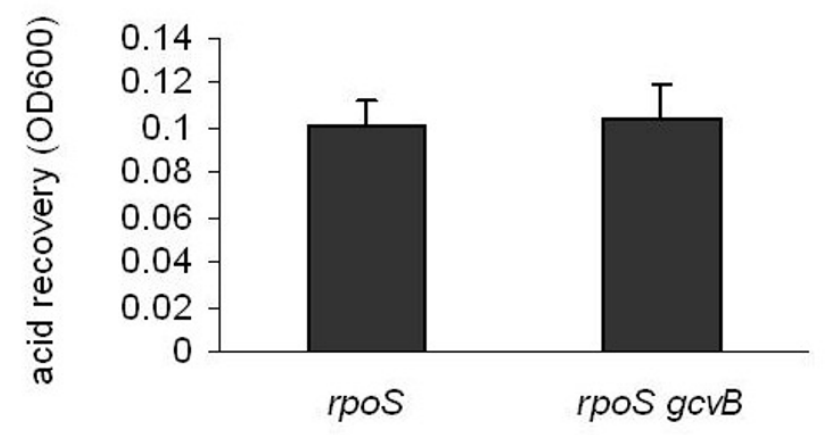

B

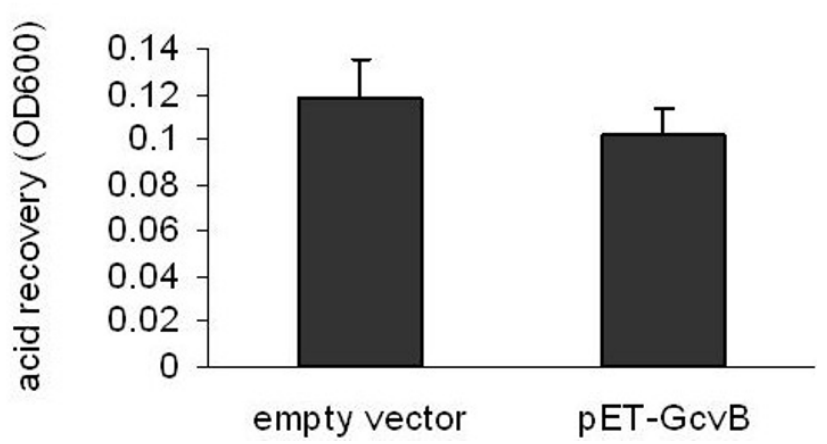

Figure 3

Effects of deleting rpoS on GcvB-mediated acid resistance. (A) Acid resistance in an rpoS mutant and an rpoS gcvB double mutant. (B) Effects of GcvB overproduction on acid resistance in an rpoS mutant. Cell cultures of $E$. coli MGI655 mutant strains were grown in LB broth ( $\mathrm{pH} 7.0)$ at $37^{\circ} \mathrm{C}$ for $5 \mathrm{hrs}$ and then acid challenged $(\mathrm{pH} 2.0)$ for 15 min. After neutralization by adding alkalinized LB ( $\mathrm{pH}$ 9.3), cells were cultured for an additional $3 \mathrm{hrs}$ before measurement of optical density at $600 \mathrm{~nm}(\mathrm{OD} 600 \mathrm{~nm})$. Error bars represent standard deviation (* $P$-value $<0.05)$.

is therefore unlikely that BarA is a potential target for GcvB. Consequently, we examined whether GcvB exerts its positive control of RpoS through Crp, H-NS or GadX. We found that deleting $g c v B$ reduced acid survival levels in an hns mutant background. This was also the case for crpor gadX-deficient strains (Fig. 5). Consistent with the knockout data, $g c v B$ overexpression increased AR levels in the hns-, crp- or gadX-deficient strains (Fig. 6). These results suggest that Hns, Crp and GadX are not involved in the GcvB-mediated regulation of rpoS expression.

$r p o S$ transcription is driven by a major promoter $r p o S p$ and two weak promoters $n l p D p 1$ and 2 [26]. To examine whether GcvB affected rpoS expression at the transcrip-
A

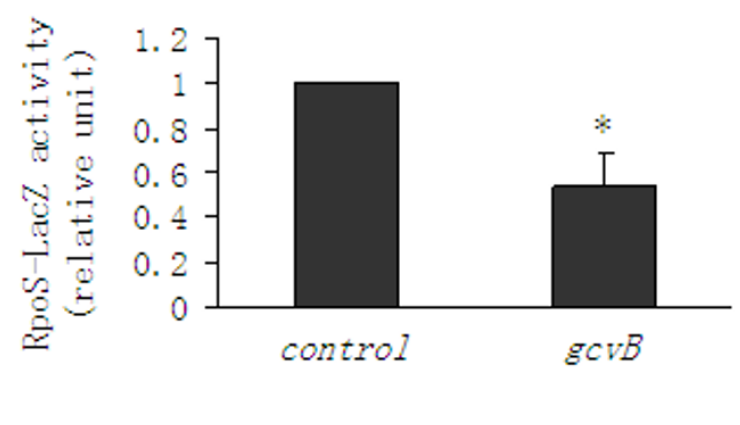

B

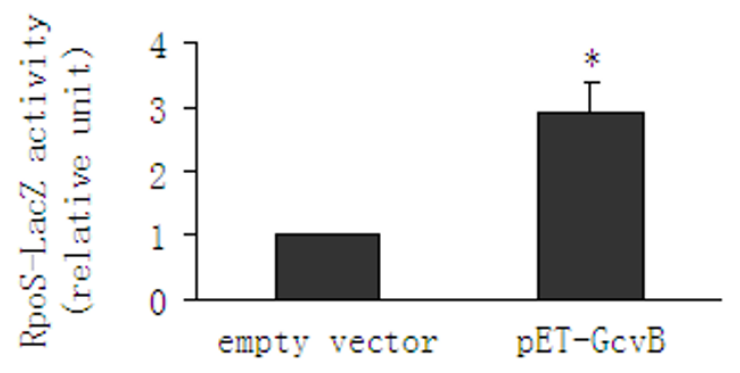

C

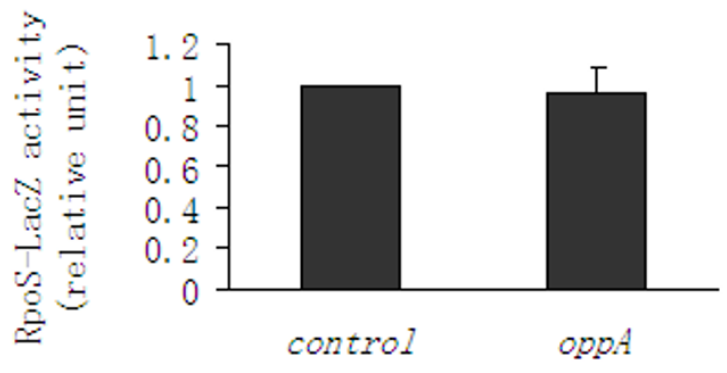

Figure 4

Effects of GcvB sncRNA and OppA protein on the expression of RpoS in a chromosomal rpoS-lacZ reporter strain. $(A)$ Effects of deleting gcvB on the expression of an rpoS-lacZ reporter gene fusion in E. coli MG 1655.

(B) Effect of GcvB overproduction on the expression of the rpoS-lacZ fusion. GcvB production was driven by its native promoter on a PET32a plasmid construct. (C) Effect of deleting oppA on the expression of the rpoS-lacZ reporter. Betagalactosidase activities due to translation of chromosomal lacZ fusions were normalized to those of MGI655 carrying an rpoS-lacZ fusion with or without an empty vector (controls). Error bars represent standard deviation (*P-value $<$ 0.05). 


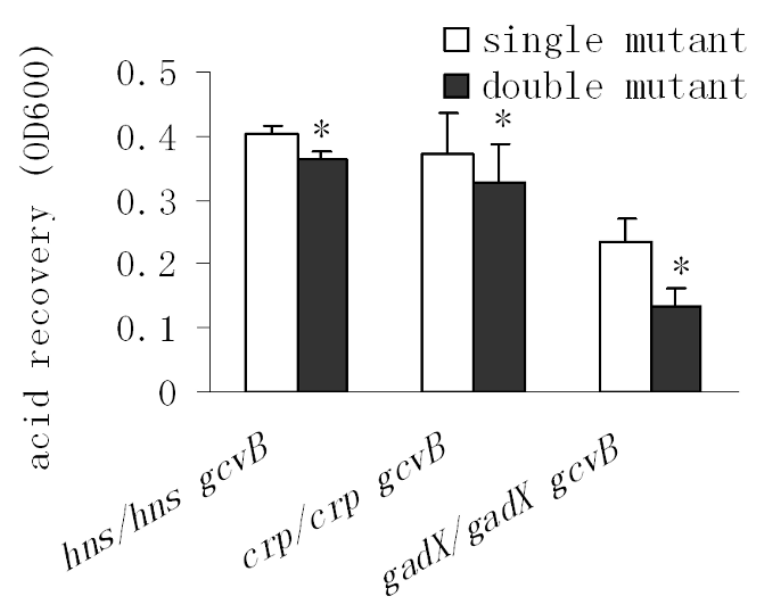

Figure 5

Effects of deleting gcvB on acid resistance in mutants lacking hns, crp or gadX. Cell cultures of E. coli MGI 655 mutant strains containing single deletions of the hns, crp or gadX genes were grown in LB broth $\left(\mathrm{pH} \mathrm{7.0)}\right.$ at $37^{\circ} \mathrm{C}$ for 5 hrs and then acid challenged $(\mathrm{pH} 2.0)$ for 15 min (gadX mutant) or $30 \mathrm{~min}$ (hns and crp mutants). After neutralization by adding alkalinized LB ( $\mathrm{pH}$ 9.3), cells were cultured for an additional 3 hrs before measurement of optical density at 600 $\mathrm{nm}(\mathrm{OD} 600 \mathrm{~nm})$. Error bars represent standard deviation (* $P$-value $<0.05)$.

tional level, we deleted the region spanning the rpoSp, $n l p D p 1$ and 2 promoter sites (Fig. 4). As removing these promoters will eliminate rpoS expression, a constitutive promoter encoded in the knockout cassette (described in Materials and Methods) was used to drive rpoS transcription in the absence of $r p o S p, n l p D p 1$ and 2 . The resulting mutant was referred to as $d_{567-1342}$. As shown in Fig. 6, replacing the native promoters of $r p o S$ with this heterologous promoter had little effect on GcvB-induced AR. These results indicate that GcvB does not regulate rpoS expression at the transcriptional level.

\section{Discussion}

In this study, we constructed a library comprising singlegene knockout mutants of seventy-nine sncRNA genes in E. coli. We used this mutant collection to identify sncRNAs involved in acid resistance (AR) within this bacterium. Consequently, we were able to identify the sncRNA GcvB as a novel regulator of AR.

DppA and OppA, which were previously identified as targets of GcvB, were shown not to be responsible for GcvBmediated AR. While the d $p p A$ deletion did not affect AR, the absence of OppA slightly improved the ability of $E$. coli to survive under extreme acid conditions. Since GcvBmediated inhibition of $o p p A$ and $d p p A$ only weakly increased AR, we reasoned that the GcvB sncRNA may be regulating AR via some other mechanistic pathway.

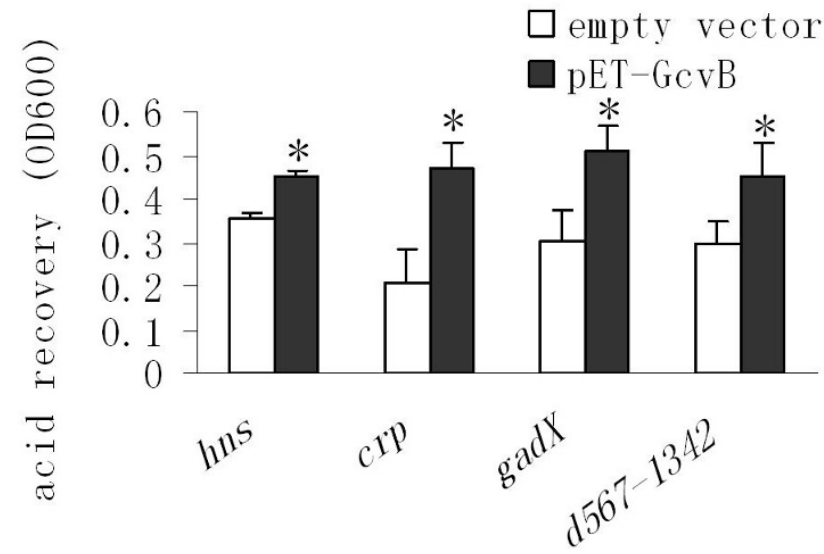

Figure 6

Effects of GcvB overexpression on acid resistance in mutants lacking hns, crp, gadX or native promoters of rpoS. The mutant lacking the native promoters of $r p o S$ was

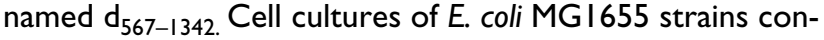
taining single deletions were grown in $\mathrm{LB}$ broth $(\mathrm{pH} 7.0)$ at $37^{\circ} \mathrm{C}$ for $5 \mathrm{hrs}$ and then acid challenged $(\mathrm{pH} 2.0)$ for 15 min (gadX mutant) or 30 min ( $h n s$ and crp mutants and $d_{567-1342}$ ). After neutralization by alkalinized LB ( $\mathrm{pH} 9.3$ ), cells were cultured for an additional $3 \mathrm{hrs}$ before measurement of optical density at $600 \mathrm{~nm}$ (OD600 nm). Error bars represent standard deviation ( $* P$-value $<0.05)$.

Upon rpoS deletion, GcvB had no influence on AR. Furthermore, we found that GcvB positively regulated $r p o S$ expression. Deleting oppA had no effect on the levels of RpoS, providing evidence that the GcvB-mediated control of RpoS does not involve the OppA protein. Also, GcvB does not regulate RpoS via H-NS, Crp, or GadX; as GcvBmediated AR was unaffected when these genes were singularly deleted. The region spanning from -567 to $-1342 \mathrm{nt}$ harbors all three of the of the rpos promoters (rposp, $n l p D p 1$ and 2). Replacing these native promoters with the constitutive promoter from a $\mathrm{Cm}$ knockout cassette had no affect on GcvB-mediated AR. This suggests that GcvB does not regulate rpoS expression at the transcriptional level.

Like GcvB, the sncRNAs DsrA and RprA also act as positive regulators of AR in E. coli. DsrA and RprA bind to the same region of the 5 leader of $r p o S$ mRNA. A sequence within this region was previously predicted to form a self-inhibitory stem loop structure that probably occludes the ShineDalgarno ribosome binding site and prevents translation $[24,27]$. DsrA $[24,27]$ and RprA $[28,29]$ bind to this stemloop structure, free the ribosome binding site, and thereby mediate rpoS translation. This region also contains RNase III cleavage sites that result in rapid decay of the rpoS transcript [30]. The base-pairing of DsrA within this region stabilizes $r p o S$ mRNA by creating an alternative RNase III cleavage site [30]. As for GcvB, it regulates rpoS expression 
by an unknown mechanism. Computational sequence analyses using the RNAhybrid server [31] have indicated that GcvB does not contain any extensive regions of sequence complementary to the $5^{\prime}$ leader of rpoS mRNA (data not shown). As such, the mechanism by which GcvB regulates rpos expression remains a subject of further investigation.

\section{Conclusion}

Here we reveal that the sncRNA GcvB positively regulates the ability of $E$. coli to survive low $\mathrm{pH}$ conditions by upregulating the expression of RpoS. Our findings provide insight into the control of AR by GcvB. However, further detailed studies will be required to investigate the precise nature of the $\mathrm{GcvB} / r$ poS interaction at the molecular level.

\section{Methods}

\section{Bacterial strains, plasmids, and growth conditions}

E. coli K12-MG1655 was used for all gene deletion and overexpression experiments. The E. coli expression vector pET32a (+) (Novagen, Madison, WI) was used for the overexpression of sncRNAs. All bacterial strains were grown at $37^{\circ} \mathrm{C}$, with shaking at $230 \mathrm{rpm}$, in Luria-Bertani (LB) medium supplemented with antibiotics when required. Bacterial growth was monitored by measuring optical density at $600 \mathrm{~nm}$ (OD600). The antibiotics ampicillin $(50 \mathrm{ug} / \mathrm{ml})$, kanamycin $(50 \mathrm{ug} / \mathrm{ml})$, and chloramphenicol $(12.5 \mathrm{ug} / \mathrm{ml})$ were used for selection.

\section{Gene deletions}

Gene deletion mutations were constructed using the $\lambda$ Red recombination system. E. coli MG1655 was transformed with plasmid pSim6 (a gift from Dr. Donald Court) [32] on which the expression of the $\lambda$ recombination proteins is induced at $42^{\circ} \mathrm{C}$. PCR fragments encompassing a loxP-cm-loxP ('floxed') chloramphenicol resistance cassette with homology (45 nt) to the regions immediately flanking each deletion locus were transformed into MG1655 harboring pSim6. After induction of $\lambda$ red, recombinants were selected for chloramphenicol $(\mathrm{cm})$ resistance, and were further verified by colony PCR.

\section{Gene cloning}

The selectable loxP-cm-loxP cassette was first inserted immediately after the stop codon of the gene of interest on the chromosome (as described above). We then used recombineering to 'clone' this gene of interest preceded by its native promoter and 'floxed' $\mathrm{cm}$ cassette downstream of the T7 promoter in a pET32a expression vector. Specifically, we PCR amplified the gene of interest (including its native promoter) and adjacent 'floxed' $\mathrm{cm}$ cassette using primers that contained homology (45 nt) to the plasmid insertion site. The PCR product and expression vector were co-transformed into DY330[12] after induction of $\lambda$ red. Recombinants were selected for chloramphenicol resistance and verified by PCR and sequencing. The native promoter drove the gene overexpression in MG1655 without induction.

\section{Construction of chromosomal lacZ translational fusions and beta-galactosidase assays}

First, the loxP-cm-loxP selectable cassette was inserted immediately after the stop codon of the lac $Z$ gene on the MG1655 chromosome using $\lambda$ red recombination (as described above). Next, the lacZ-loxP-cm-loxP cassette was PCR amplified and inserted (in frame) immediately prior to the stop codon of the target gene on the chromosome. The inserted lac $Z$ fragment started from the $8^{\text {th }}$ codon of lacZ gene and co-transcribed and -translated with the fused genes. The expression of gene-lac $Z$ fusions was quantified using a beta-galactosidase assay kit from Thermo Fisher Scientific (Rockford, Illinois, USA).

\section{Acid resistance assay}

Overnight cultures were diluted 1:50 and incubated at $37^{\circ} \mathrm{C}$ for 5 hrs in LB medium. 3 volumes of acidified LB medium ( $\mathrm{pH}$ 1.9) was then added to 1 volume of the cell culture so that the final $\mathrm{pH}$ value was approximately 2.0. The acid challenge was maintained for $15 \mathrm{~min}$ for cells that were acid sensitive, and $30 \mathrm{~min}$ for acid-resistant cells. The acid challenge was stopped by adding 3 volumes of alkalinized LB medium (pH9.3), making the final $\mathrm{pH}$ value approximately 7.0. For samples that displayed similar growth rates, the neutralized cell cultures were allowed to grow for $3 \mathrm{hrs}$ following the acid challenge, and acid survival levels were determined by measurement of optical density at $600 \mathrm{~nm}$ (OD600, termed acid recovery). When samples with different growth rates were examined, cell viability was determined by plate counts and percentage acid survival was calculated as the number of number of colony forming units (cfu) remaining after the acid treatment divided by the initial cfu at time zero.

\section{Statistical analysis}

Two-tailed paired t-tests were used for the comparison of means obtained from the beta-galactosidase and acid survival assays. $P$ values of $<0.05$ were considered statistically significant.

\section{Abbreviations}

AR: acid resistance; sncRNA: small noncoding RNA; UTR: unstranslated region.

\section{Authors' contributions}

$\mathrm{YJ}$ planned and designed the experiments, performed the experiments and the data analysis, wrote the main draft of the paper, and generated the figures. RW conceived of the study and helped to draft the manuscript. $\mathrm{AD}$ aided in the experimental design and in editing the manuscript. JH provided guidance over the entire project, and aided in 
data analysis and editing of the manuscript. All authors read and approved the final manuscript.

\section{Additional material}

\section{Additional file 1}

Small noncoding RNAs (sncRNA) that were selected for construction of a single-sncRNA gene knockout library within E. coli K12-

MG1655. This table summarizes positions and functions of the small noncoding RNAs that were included in a single-sncRNA gene knockout library within E. coli K12-MG1655.

Click here for file

[http://www.biomedcentral.com/content/supplementary/14712164-10-165-S1.doc]

\section{Acknowledgements}

We thank D.L. Court for providing plasmid pSim6 for the recombineering experiments. This work was supported by HKU CRCG Seed Funding Programme for Basic Research and by a grant (HKU 7485/06M) from the Research Grant Council of Hong Kong to JDH. AD acknowledges support from the PROBACTYS programme, grant CT-2006-029104 in an effort to define genes essential for the construction of a synthetic cell.

\section{References}

I. Castanie-Cornet MP, Penfound TA, Smith D, Elliott JF, Foster JW Control of acid resistance in Escherichia coli. J Bacteriol 1999 I 8 I ( I I ):3525-3535.

2. Hersh BM, Farooq FT, Barstad DN, Blankenhorn DL, Slonczewski JL: A glutamate-dependent acid resistance gene in Escherichia coli. J Bacteriol 1996, I 78(1 3):3978-398I.

3. Lin J, Smith MP, Chapin KC, Baik HS, Bennett GN, Foster JW: Mechanisms of acid resistance in enterohemorrhagic Escherichia coli. Appl Environ Microbiol 1996, 62(9):3094-3100.

4. Sledjeski DD, Gupta A, Gottesman S: The small RNA, DsrA, is essential for the low temperature expression of RpoS during exponential growth in Escherichia coli. EMBO J 1996, I 5(I5):3993-4000.

5. Lease RA, Smith D, McDonough K, Belfort M: The small noncoding DsrA RNA is an acid resistance regulator in Escherichia coli. J Bacteriol 2004, 186(18):6179-6/85.

6. Opdyke JA, Kang JG, Storz G: GadY, a small-RNA regulator of acid response genes in Escherichia coli. J Bacteriol 2004, I 86(20):6698-6705

7. Tramonti A, De Canio M, De Biase D: GadX/GadW-dependent regulation of the Escherichia coli acid fitness island: transcriptional control at the gadY-gadW divergent promoters and identification of four novel 42-bp GadX/GadW-specific binding sites. Mol Microbiol 2008, 70(4):965-982.

8. Griffiths-Jones S, Moxon S, Marshall M, Khanna A, Eddy SR, Bateman $A$ : Rfam: annotating non-coding RNAs in complete genomes. Nucleic Acids Res 2005:D I 21-I 24.

9. Vallenet D, Labarre L, Rouy Z, Barbe V, Bocs S, Cruveiller S, Lajus A, Pascal G, Scarpelli C, Medigue C: MaGe: a microbial genome annotation system supported by synteny results. Nucleic Acids Res 2006, 34(I):53-65.

10. Vogel J, Bartels V, Tang TH, Churakov G, Slagter-jager JG, Huttenhofer A, Wagner EG: RNomics in Escherichia coli detects new SRNA species and indicates parallel transcriptional output in bacteria. Nucleic Acids Res 2003, 3 I (22):6435-6443.

II. Ellis HM, Yu D, DiTizio T, Court DL: High efficiency mutagenesis, repair, and engineering of chromosomal DNA using singlestranded oligonucleotides. Proc Natl Acad Sci USA 200I, 98(I2):6742-6746.

12. Yu D, Ellis HM, Lee EC, Jenkins NA, Copeland NG, Court DL: An efficient recombination system for chromosome engineer- ing in Escherichia coli. Proc Natl Acad Sci USA 2000, 97(II):5978-5983.

13. Urbanowski ML, Stauffer LT, Stauffer GV: The gcvB gene encodes a small untranslated RNA involved in expression of the dipeptide and oligopeptide transport systems in Escherichia coli. Mol Microbiol 2000, 37(4):856-868.

14. Urban JH, Vogel J: Translational control and target recognition by Escherichia coli small RNAs in vivo. Nucleic Acids Res 2007, 35(3): $1018-1037$

15. Sharma CM, Darfeuille F, Plantinga TH, Vogel J: A small RNA regulates multiple ABC transporter mRNAs by targeting C/Arich elements inside and upstream of ribosome-binding sites. Genes Dev 2007, 2 I (2I):2804-28I7.

16. Tramonti A, Visca P, De Canio M, Falconi M, De Biase D: Functional characterization and regulation of gad $X$, a gene encoding an AraC/XyIS-like transcriptional activator of the Escherichia coli glutamic acid decarboxylase system. J Bacteriol 2002, I 84(I0):2603-26I3.

17. Sayed AK, Odom C, Foster JW: The Escherichia coli AraC-family regulators $\mathrm{GadX}$ and $\mathrm{GadW}$ activate gadE, the central activator of glutamate-dependent acid resistance. Microbiology 2007, I 53 (Pt 8):2584-2592.

18. Small P, Blankenhorn D, Welty D, Zinser E, Slonczewski JL: Acid and base resistance in Escherichia coli and Shigella flexneri: role of rpoS and growth pH. J Bacteriol 1994, I76(6): 1729-1737.

19. Mika F, Hengge R: A two-component phosphotransfer network involving ArcB, ArcA, and RssB coordinates synthesis and proteolysis of sigmaS (RpoS) in E. coli. Genes Dev 2005, 19(22):2770-278|.

20. Lange R, Hengge-Aronis R: The cellular concentration of the sigma $S$ subunit of RNA polymerase in Escherichia coli is controlled at the levels of transcription, translation, and protein stability. Genes Dev 1994, 8(13):1600-1612.

21. Mukhopadhyay S, Audia JP, Roy RN, Schellhorn HE: Transcriptional induction of the conserved alternative sigma factor RpoS in Escherichia coli is dependent on BarA, a probable two-component regulator. Mol Microbiol 2000, 37(2):37I-38I.

22. Hommais F, Krin E, Coppee JY, Lacroix C, Yeramian E, Danchin A, Bertin P: GadE (YhiE): a novel activator involved in the response to acid environment in Escherichia coli. Microbiology 2004, I 50(Pt I):6I-72.

23. Brescia CC, Kaw MK, Sledjeski DD: The DNA binding protein $\mathbf{H}$ NS binds to and alters the stability of RNA in vitro and in vivo. J Mol Biol 2004, 339(3):505-5I4.

24. Lease RA, Cusick ME, Belfort M: Riboregulation in Escherichia coli: DsrA RNA acts by RNA:RNA interactions at multiple loci. Proc Natl Acad Sci USA 1998, 95(2I): | 2456-|246I.

25. Zhang A, Altuvia S, Tiwari A, Argaman L, Hengge-Aronis R, Storz G: The OxyS regulatory RNA represses rpoS translation and binds the Hfq (HF-I) protein. EMBO J 1998, I7(20):606I-6068.

26. Lange R, Fischer D, Hengge-Aronis R: Identification of transcriptional start sites and the role of ppGpp in the expression of rpoS, the structural gene for the sigma S subunit of RNA polymerase in Escherichia coli. J Bacteriol 1995, I77(16):4676-4680.

27. Majdalani N, Cunning C, Sledjeski D, Elliott T, Gottesman S: DsrA RNA regulates translation of RpoS message by an anti-antisense mechanism, independent of its action as an antisilencer of transcription. Proc Natl Acad Sci USA 1998, 95(2I): 12462-12467.

28. Majdalani N, Hernandez D, Gottesman S: Regulation and mode of action of the second small RNA activator of RpoS translation, RprA. Mol Microbiol 2002, 46(3):8I3-826.

29. Updegrove T, Wilf N, Sun X, Wartell RM: Effect of Hfq on RprArpoS mRNA pairing: Hfq-RNA binding and the influence of the $5^{\prime}$ rpos mRNA leader region. Biochemistry 2008, 47(43): I | |84-I I I95.

30. Resch A, Afonyushkin T, Lombo TB, McDowall KJ, Blasi U, Kaberdin VR: Translational activation by the noncoding RNA DsrA involves alternative RNase III processing in the rpoS 5'leader. RNA 2008, I4(3):454-459.

31. Kruger J, Rehmsmeier M: RNAhybrid: microRNA target prediction easy, fast and flexible. Nucleic Acids Res 2006:W45I-454.

32. Datta S, Costantino N, Court DL: A set of recombineering plasmids for gram-negative bacteria. Gene 2006, 379:109-1 I5. 